\title{
INCOMPRESSIBILITY ESTIMATES FOR THE LAUGHLIN PHASE, PART II
}

\author{
NICOLAS ROUGERIE AND JAKOB YNGVASON
}

\begin{abstract}
We consider fractional quantum Hall states built on Laughlin's original $N$ body wave-functions, i.e., they are of the form holomorphic $\times$ gaussian and vanish when two particles come close, with a given polynomial rate. Such states appear naturally when looking for the ground state of $2 \mathrm{D}$ particles in strong magnetic fields, interacting via repulsive forces and subject to an external potential due to trapping and/or disorder. We prove that all functions in this class satisfy a universal local density upper bound, in a suitable weak sense. Such bounds are useful to investigate the response of fractional quantum Hall phases to variations of the external potential. Contrary to our previous results for a restricted class of wave-functions, the bound we obtain here is not optimal, but it does not require any additional assumptions on the wave-function, besides analyticity and symmetry of the pre-factor modifying the Laughlin function.
\end{abstract}

\section{Contents}

1. Introduction

1.1. Background

1.2. Main result

1.3. Method of proof: The plasma analogy

2. Estimates for ground state configurations

2.1. Density Upper bounds

2.2. Separation of points in the ground state

3. Applications to Gibbs states at small temperature

3.1. Small temperature limit

3.2. Conclusion of the proof of Theorem 1.1

References

\section{Introduction}

1.1. Background. The fractional quantum Hall effect (FQHE) [20, 6] is a signature of the transition to a strongly correlated quantum fluid in the ground state of a $2 \mathrm{D}$ electron gas submitted to a strong perpendicular magnetic field. The best known of these states is described by Laughlin's wave function [7]

$$
\Psi_{\text {Lau }}\left(z_{1}, \ldots, z_{N}\right)=c_{\text {Lau }} \prod_{1 \leq i<j \leq N}\left(z_{i}-z_{j}\right)^{\ell} e^{-\sum_{j=1}^{N}\left|z_{j}\right|^{2} / 2} .
$$

Date: November 3, 2014. 
Here, $z_{1}, \ldots, z_{N}$ are the coordinates, identified with complex numbers, of $N$ quantum particles in 2D. Since electrons are fermions, the positive integer $\ell$ should be an odd number for symmetry reasons. If $\ell=1$ the function (1.1) is simply a Slater determinant, so for interacting fermions $\ell \geq 3$ is the important case. We shall also consider the case where $\ell$ is even so the wave function describes bosonic particles; this case is in particular relevant for ultra-cold Bose gases with short range repulsive interactions in rapidly rotating traps [9, 16, 17].

The form of the function (1.1) is dictated by two requirements:

- The function should belong to the lowest Landau level of a magnetic Laplacian, whence the form "holomorphic $\times$ gaussian". We have chosen units so that the width of the gaussian is fixed, i.e. does not depend explicitly on the magnetic field strength or the rotation speed.

- The particles want to avoid one another in order to reduce as much as possible the interaction energy for a given repulsive potential. The holomorphic factor in (1.1) is thus chosen to vanish along the diagonals $z_{i}=z_{j}$ with a chosen polynomial rate $\ell \in \mathbb{N}$.

The constant $c_{\text {Lau }}$ normalizes the function in $L^{2}\left(\mathbb{R}^{2 N}\right)$ to make it a state vector that is a promising candidate for approximating the ground state of $2 \mathrm{D}$ quantum particles in a strong magnetic field, with strong repulsive interaction between the particles. More generally, in an external potential, natural candidates live in the space

$$
\mathcal{L}_{\ell}^{N}:=\left\{\Psi \in L^{2}\left(\mathbb{R}^{2 N}\right): \Psi\left(z_{1}, \ldots, z_{N}\right)=\Psi_{\text {Lau }}\left(z_{1}, \ldots, z_{N}\right) F\left(z_{1}, \ldots, z_{N}\right), F \in \mathcal{B}^{N}\right\} .
$$

Here $\mathcal{B}^{N}$ denotes the $N$-body bosonic Bargmann space

$$
\mathcal{B}^{N}:=\left\{F \text { holomorphic and symmetric } \mid F\left(z_{1}, \ldots, z_{N}\right) e^{-\sum_{j=1}^{N}\left|z_{j}\right|^{2} / 2} \in L^{2}\left(\mathbb{R}^{2 N}\right)\right\} .
$$

Note that the symmetry constraint on $F$ implies that $\mathcal{L}_{\ell}^{N}$ is an appropriate function space for fermions (resp. bosons) if $\ell$ is odd (resp. even). We thus treat both type of particles in the same way and are not able to exploit the effect of statistics beyond that of fixing the parity of $\ell$.

The functions in $\mathcal{L}_{\ell}^{N}$ are annihilated by two-body contact interactions [21, 12], and for $\ell=2$ this space is exactly the kernel $\operatorname{Ker}\left(\mathcal{I}_{N}\right)$ of the contact interaction Hamiltonian

$$
\mathcal{I}_{N}:=\sum_{1 \leq i<j \leq N} \delta_{i j}
$$

acting on the $N$-body bosonic lowest Landau level by the prescription

$$
\delta_{i, j}\left(F\left(z_{1}, \ldots, z_{N}\right) e^{-\sum_{j=1}^{N}\left|z_{j}\right|^{2} / 2}\right)=F\left(z_{1}, \ldots, \frac{z_{i}+z_{j}}{2}, \ldots, \frac{z_{i}+z_{j}}{2}, \ldots, z_{N}\right) e^{-\sum_{j=1}^{N}\left|z_{j}\right|^{2} / 2} .
$$

We refer to our previous papers [17, 16] and in particular [18] for more background and references. In particular, our notion of "incompressibility estimates" is explained in 18 and we recall it briefly in the next two paragraphs.

The variational space $\mathcal{L}_{\ell}^{N}$ is of course huge and it is of importance in practice to be able to determine which state will emerge as a natural candidate for the ground state in a given situation. All states in $\mathcal{L}_{\ell}^{N}$ have by definition the same magnetic kinetic energy, and since they all vanish with (at least) the same rate $\left|z_{i}-z_{j}\right|^{\ell}$ along the diagonals 
of configuration space it is natural to expect that they will also have roughly the same (small) interaction energy for a wide class of interaction potentials. The emergence of one particular state as a candidate for a ground state must thus be a consequence of effects so far neglected: in quantum Hall system: 1, these come mostly from the energetic contribution of external fields, due to trapping/and or disorder in a given sample. In other words, it seems reasonable that the emergence of a particular state should follow from the minimization of some reduced energy functional on $\mathcal{L}_{\ell}^{N}$.

To be able to tackle such a problem, it is useful to know what properties (normalized) wave functions of $\mathcal{L}_{\ell}^{N}$ have in common. One such property should be an incompressibility, or rigidity, bound: there is good reason to believe that a $\Psi \in \mathcal{L}_{\ell}^{N}$ with $\|\Psi\|_{L^{2}}=1$ satisfies

$$
\rho_{\Psi} \leq \frac{1}{\pi \ell N}
$$

at least in a suitable weak sense, where

$$
\rho_{\Psi}(z):=\int_{\mathbb{R}^{2(N-1)}}\left|\Psi\left(z, z_{2}, \ldots, z_{N}\right)\right|^{2} d z_{2} \ldots d z_{N}
$$

is the one-body probability density of $\Psi$. Note that (1.4) is in fact optimal in the sense that it is saturated for certain choices of $\Psi$, in particular the Laughlin functions themselves, in the large $N$ limit, see [16. Note, however, that (1.4) is not expected to hold point-wise for finite $N$ [2].

This paper is devoted to the proof of estimates in this spirit. It is a sequel to [18] where we introduced a suitable weak formulation of (1.4) and proved that it is satisfied for $\Psi$ of the particular form

$$
\Psi\left(z_{1}, \ldots, z_{N}\right)=\Psi_{\text {Lau }}\left(z_{1}, \ldots, z_{N}\right) F\left(z_{1}, \ldots, z_{N}\right), F \in \mathcal{V}_{2}^{D},\|\Psi\|_{L^{2}}=1,
$$

where (deg denotes the degree of a polynomial) $F$ belongs to the set

$$
\begin{array}{r}
\mathcal{V}_{2}^{D}=\left\{F \in \mathcal{B}^{N}: \text { there exist }\left(f_{1}, f_{2}\right) \in \mathcal{B} \times \mathcal{B}^{2}, \operatorname{deg}\left(f_{1}\right) \leq D N, \operatorname{deg}\left(f_{2}\right) \leq D,\right. \\
\left.F\left(z_{1}, \ldots, z_{N}\right)=\prod_{j=1}^{N} f_{1}\left(z_{j}\right) \prod_{1 \leq i<j \leq N} f_{2}\left(z_{i}, z_{j}\right)\right\} .
\end{array}
$$

In [18] it is argued that the bound should hold more generally if the pre-factor $F$ is a symmetrized product of functions that depend only on a fixed number $n$ of variables with $n \ll N$.

The main result of the present paper is a (weak version of a) bound of the form (1.4) without any a priori assumption on $F$ except analyticity and symmetry. We prove that, in a sense made precise in Theorem 1.1 below,

$$
\rho_{\Psi} \leq \frac{4}{\pi \ell N}
$$

for any $\Psi \in \mathcal{L}_{\ell}^{N}$ with $\|\Psi\|_{L^{2}}=1$. Note that the constant on the right-hand side of (1.7) is four times the one in (1.4).

In brief, we prove in the present paper incompressibility bounds for all functions in $\mathcal{L}_{\ell}^{N}$ but with a worse constant than the (presumably optimal) one that was derived for a

\footnotetext{
${ }^{1}$ And also in the rotating Bose gases suggested to mimic them, see [18, and references therein.
} 
restricted family of wave functions in [18. This difference is due to the different methods employed. It remains an open problem to bridge the gap between [18, and the present paper by proving the optimal bound without a priori assumptions on $F$.

1.2. Main result. As discussed at length in [18, our incompressibility estimates are formulated in terms of the minimum energy that one can achieve amongst states in $\mathcal{L}_{\ell}^{N}$ feeling an external potential which lives on the scale of the Laughlin state, i.e. over lengths of order $\sqrt{N}$. This amounts to considering the energy functional

$$
\mathcal{E}_{N}[\Psi]=(N-1) \int_{\mathbb{R}^{2}} V(\mathbf{x}) \rho_{\Psi}(\sqrt{N-1} \mathbf{x})
$$

where $V$ is a given trapping potential, $\Psi \in \mathcal{L}_{\ell}^{N}$ and $\rho_{\Psi}$ is the corresponding one-particle probability density (1.5). Note the choice of normalization: we have set

$$
\int_{\mathbb{R}^{2}}(N-1) \rho_{\Psi}(\sqrt{N-1} \mathbf{x}) d \mathbf{x}=1 .
$$

We shall be concerned with the large $N$ behavior of the ground state energy

$$
E(N):=\inf \left\{\mathcal{E}_{N}\left[\Psi_{F}\right], \Psi_{F} \in \mathcal{L}_{\ell}^{N},\|\Psi\|_{L^{2}}=1\right\} .
$$

The following is our main result:

Theorem 1.1 (Unconditional incompressibility for $\mathcal{L}_{\ell}^{N}$ ). Let $V \in C^{2}\left(\mathbb{R}^{2}\right)$ be increasing at infinity in the sense that

$$
\min _{|x| \geq R} V(\mathbf{x}) \rightarrow \infty \text { for } R \rightarrow \infty .
$$

Define the corresponding "bathtub energy" (cf. [10, Theorem 1.14]) by

$$
E_{V}(\ell / 4):=\inf \left\{\int_{\mathbb{R}^{2}} V \rho \mid \rho \in L^{1}\left(\mathbb{R}^{2}\right), 0 \leq \rho \leq \frac{4}{\pi \ell}, \int_{\mathbb{R}^{2}} \rho=1\right\} .
$$

Then

$$
\liminf _{N \rightarrow \infty} E(N) \geq E_{V}(\ell / 4) .
$$

This is a weak version of (1.7) in the sense that a universal lower bound to the energy $\mathcal{E}_{N}\left[\Psi_{F}\right]$ can be computed just as if (1.7) would hold, for a large class of one-body potentials. The notation $E_{V}(\ell / 4)$ corresponds to the convention in [18, Theorem 2.1]. We conjecture that the lower bound (1.12) can be improved to

$$
\liminf _{N \rightarrow \infty} E(N) \geq E_{V}(\ell)
$$

but this is out of reach with the method we use here. In [18, Theorem 2.1] we proved a conditional version of such a lower bound using a different method, i.e. we showed that (1.13) holds under the a priori assumption (reasonable but not rigorously justified) that it suffices to consider correlation factors $F$ belonging to (1.6). Note that $E_{V}(\ell)$ is clearly an increasing function of $\ell$. For the homogeneous potential $V(x)=|x|^{s}$, it is easy to see that

$$
E_{V}(\ell)=\frac{2}{s+2} \ell^{s / 2} \text {. }
$$


Another natural conjecture is that the minimum energy $E(N)$ can always be achieved in the Laughlin phase of functions of the form

$$
\Psi\left(z_{1}, \ldots, z_{N}\right)=c_{f_{1}} \Psi_{\text {Lau }}\left(z_{1}, \ldots, z_{N}\right) \prod_{j=1}^{N} f_{1}\left(z_{j}\right), \quad\|\Psi\|_{L^{2}}=1,
$$

that is functions that do not contain any correlation factors beside the Laughlin state itself. In view of [18, Corollary 2.3], this would follow at least for some special radial potentials from the optimal bound (1.13). This conjecture means informally that it is never favorable to leave the Laughlin phase by adding more correlations, whatever the one-body potential.

1.3. Method of proof: The plasma analogy. As in our previous works [16, 17, 18, the starting point is Laughlin's plasma analogy, which is reminiscent of the log-gas analogy in random matrix theory [1, 3, 4, 5, 11. The crucial observation [7, 8] is that the absolute square of the wave-function (1.1) can be regarded as the Gibbs state of a 2D Coulomb gas (one-component plasma). Following our approach in [18 we generalize this idea to map any state of $\mathcal{L}_{\ell}^{N}$ to a Gibbs state of a classical Hamiltonian.

Let us consider a state

$$
\Psi_{F}=c_{F} \Psi_{\text {Lau }}\left(z_{1}, \ldots, z_{N}\right) F\left(z_{1}, \ldots, z_{N}\right)
$$

where $\Psi_{\text {Lau }}$ is the Laughlin state (1.1), $F$ is holomorphic and symmetric and $c_{F}$ is a normalization factor. The idea is simply to write

$$
\left|\Psi_{F}\right|^{2}=c_{F}^{2} \exp \left(-2 \log \left|\Psi_{\text {Lau }}\right|-2 \log |F|\right)
$$

and interpret $2 \log \left|\Psi_{\text {Lau }}\right|+2 \log |F|$ as a classical Hamilton function. One of the reasons why this is an effective procedure is that we can take advantage of the good scaling properties of $\left|\Psi_{\text {Lau }}\right|^{2}$ to first change variables and obtain a classical Gibbs states with mean-field two-body interactions and small effective temperature. Specifically, we define

$$
\mu_{N}(Z):=(N-1)^{N}\left|\Psi_{F}(\sqrt{N-1} Z)\right|^{2}=\frac{1}{\mathcal{Z}_{N}} \exp \left(-\frac{1}{T} H_{N}(Z)\right)
$$

where $\mathcal{Z}_{N}$ ensures normalization of $\mu_{N}$ in $L^{1}\left(\mathbb{R}^{2 N}\right)$,

$$
T=\frac{1}{N}
$$

and the classical Hamiltonian is of the form

$$
H_{N}(Z)=\sum_{j=1}^{N}\left|z_{j}\right|^{2}+\frac{2 \ell}{N-1} \sum_{1 \leq i<j \leq N} w\left(z_{i}-z_{j}\right)+\frac{1}{N-1} W(Z) .
$$

We have here written

for the 2D Coulomb kernel and defined

$$
w(z):=-\log |z|
$$

$$
W(Z):=-2 \log |F(\sqrt{N-1} Z)| .
$$

The $n$-th marginal of $\mu_{N}$ is defined as

$$
\mu_{N}^{(n)}\left(z_{1}, \ldots, z_{n}\right):=\int_{\mathbb{R}^{2 N-n}} \mu_{N}\left(z_{1}, \ldots, z_{N}\right) d z_{n+1} \ldots d z_{N} .
$$


We are ultimately interested in an incompressibility bound of the form (this is (1.4) after changing length and density units as in (1.15))

$$
\mu_{N}^{(1)} \lesssim \frac{\text { const. }}{\pi \ell}
$$

in an appropriate weak sense, independently of the details of $W$.

The function (1.18) can be rather intricate and represents in general a genuine $N$-body interaction term of the Hamiltonian (1.16). The only thing we know a priori about $W$ is that it is superharmonic in each of its variables:

$$
-\Delta_{z_{j}} W \geq 0 \quad \forall j=1 \ldots N
$$

which follows from the fact that $F$ is holomorphic. As explained in [18, under the additional assumption that $F$ belongs to $\mathcal{V}_{2}^{D}$, it is possible to regard $W$ as a few-body interaction in a mean-field like scaling. Bounds of the form (1.19) where in [18] shown to follow from a mean-field approximation for $\mu_{N}$ : We first justified that an ansatz of the form

$$
\mu_{N} \approx \rho^{\otimes N}, \quad \rho \in L^{1}\left(\mathbb{R}^{2}\right)
$$

is an effective ansatz for deriving density bounds on $\mu_{N}^{(1)}$. It then followed from superharmonicity of $W$ that the appropriate $\rho$ must satisfy the desired incompressibility bound (1.19), with the optimal constant 1. The difficult part in this approach was the justification of the ansatz (1.21). In the general case there does not seem to be any reason why (1.21) should be a good approximation, and a new strategy is called for.

There is, in fact, another way to bound the one-particle density in ground states of Coulomb systems without using any mean-field approximation. The argument is due to Lieb (unpublished), and variants thereof have been used recently in [13, 14, 15. It is based solely on some properties of the Coulomb kernel and general superharmonicity arguments, so that it can be adapted to Hamiltonians of the form (1.16). Our strategy to prove Theorem 1.1 is then the following:

- We adapt Lieb's argument to obtain a bound on the minimal separation of points in the ground state configurations. This implies a local density upper bound at the level of the ground state, but not yet the Gibbs state (1.15).

- We exploit the fact that (1.15) is a Gibbs state for $H_{N}$ with small temperature $T \rightarrow 0$ in the limit $N \rightarrow \infty$. It is thus reasonable to expect the density bound on the ground state to also apply to the Gibbs state.

- More precisely, appropriate upper and lower bounds to the free-energy $-T \log \mathcal{Z}_{N}$ confirm that it is close to the ground state energy of $H_{N}$ in the limit $T \rightarrow 0$. Applying these bounds to a suitably perturbed Hamiltonian gives the desired estimates on the density by a Feynman-Hellmann type argument.

This method has the merit of yielding weak density upper bounds, under the sole assumption that $F$ is holomorphic and symmetric. It can, however, not give the expected optimal bound (1.13) since it is based on a minimal distance estimate for ground state configurations: Obtaining the optimal constant with this method would be basically like computing the minimal distance between points in a Coulomb system, i.e., solving the crystallization problem for repulsive 2D Coulomb systems, a notoriously hard question (see [13, 19, 14, 15] for recent progress). 
The rest of the paper is devoted to the proof of Theorem 1.1. Section 2 contains the analysis of the ground state of the classical Hamiltonian (1.16). Adaptations to Gibbs states at small temperature are given in Section 3.1. We come back to the original problem and conclude the proof in Section 3.2.

Acknowledgments. N.R. thanks the Erwin Schrödinger Institute, Vienna, for its hospitality. We received financial support from the ANR (project Mathostaq, ANR-13-JS010005-01) and the Austrian Science Fund (FWF) under project P 22929-N16.

\section{Estimates For Ground State CONFigurations}

In this section we discuss density bounds for the ground state configurations of $H_{N}$, i.e., for configurations of points $\left(z_{1}, \ldots, z_{N}\right)=Z_{N} \in \mathbb{R}^{2 N}$ minimizing the function (1.16). These density bounds will be derived from bounds on the minimal distance $\min _{i \neq j} \operatorname{dist}\left(z_{i}, z_{j}\right)$ as we explain first in Section 2.1. The lower bound on the minimal distance is then proved in Section 2.2,

2.1. Density Upper bounds. Since we shall later use a Feynman-Hellmann argument, we need to discuss, besides $H_{N}$, the perturbed Hamiltonian

$$
H_{N}^{\varepsilon}\left(Z_{N}\right)=\sum_{j=1}^{N}\left(\left|z_{j}\right|^{2}+\varepsilon U\left(z_{j}\right)\right)+\frac{2 \ell}{N-1} \sum_{1 \leq i<j \leq N} w\left(z_{i}-z_{j}\right)+\frac{1}{N-1} W(Z)
$$

where $U \in C^{2}\left(\mathbb{R}^{2}\right)$ is a uniformly bounded function with uniformly bounded derivatives up to second order. To understand the main point of the argumentation, it is sufficient to think of the case $\varepsilon=0$, however. We shall prove the following, which is reminiscent of previous results proved in [13, 14, 15] in the case $W \equiv 0$.

Proposition 2.1 (Separation of points in a ground state configuration). Let $Z_{N}^{\varepsilon}=\left(z_{1}^{\varepsilon}, \ldots, z_{N}^{\varepsilon}\right)$ be a ground state configuration for $H_{N}^{\varepsilon}$. Then, for $\varepsilon$ small enough,

$$
\min _{i \neq j} \operatorname{dist}\left(z_{i}^{\varepsilon}, z_{j}^{\varepsilon}\right) \geq \sqrt{\frac{\ell}{N-1}}\left(1-4 \varepsilon^{1 / 2}\|\Delta U\|_{L^{\infty}}^{1 / 2}\right) .
$$

To see that this indeed provides an incompressibility bound of the form (1.19) at the level of the ground state, we state the

Corollary 2.2 (Weak incompressibility bound for the ground state). Let $Z_{N}^{\varepsilon}=\left(z_{1}^{\varepsilon}, \ldots, z_{N}^{\varepsilon}\right)$ be a ground state configuration for $H_{N}^{\varepsilon}$ and

$$
\rho_{0}^{\varepsilon}(z)=\frac{1}{N} \sum_{j=1}^{N} \delta\left(z-z_{j}^{\varepsilon}\right)
$$

be the corresponding 1-particle density (empirical measure). There exists a $\tilde{\rho}_{0}^{\varepsilon} \in L^{\infty}\left(\mathbb{R}^{2}\right)$ satisfying

$$
\int_{\mathbb{R}^{2}} \tilde{\rho}_{0}^{\varepsilon}=1
$$

and

$$
\tilde{\rho}_{0}^{\varepsilon} \leq \frac{4}{\pi \ell}\left(1+8 \varepsilon^{1 / 2}\|\Delta U\|_{L^{\infty}}^{1 / 2}\right)\left(1-N^{-1}\right)
$$


for $\varepsilon$ small enough, such that for any $f \in C^{1}\left(\mathbb{R}^{2}\right)$ we have

$$
\left|\int_{\mathbb{R}^{2}}\left(\rho_{0}^{\varepsilon}-\tilde{\rho}_{0}^{\varepsilon}\right) f\right| \leq C N^{-1 / 2}\|\nabla f\|_{L^{\infty}}
$$

in the limit $N \rightarrow \infty$, with a constant $C \sim \ell^{1 / 2}$.

Proof. The obvious choice is to define

$$
\tilde{\rho}_{0}^{\varepsilon}(z)=\frac{1}{N} \sum_{j=1}^{N} \frac{1}{\pi L^{2}} \mathbb{1}_{z \in B\left(z_{j}^{\varepsilon}, L\right)}
$$

where $\mathbb{1}_{z \in B\left(z_{j}^{\varepsilon}, L\right)}$ is the characteristic function of the ball $B\left(z_{j}^{\varepsilon}, L\right)$ with center $z_{j}$ and radius $L$, with

$$
L=\frac{1}{2} \sqrt{\frac{\ell}{N-1}}\left(1-4 \varepsilon^{1 / 2}\|\Delta U\|_{L^{\infty}}^{1 / 2}\right) .
$$

Then (2.4) holds because (2.2) ensures that the balls $B\left(z_{j}^{\varepsilon}, L\right)$ do not overlap, and (2.5) is obvious, with $C \sim \ell^{1 / 2}$ since the radius of the ball is $O(\sqrt{\ell / N})$.

2.2. Separation of points in the ground state. To prove Proposition 2.1 we use the fact that any point in a minimizing configuration must sit at the minimum of the potential generated by all the other points plus the external potential. To simplify the notation we drop the $\varepsilon$ superscripts on the points $z_{j}^{\varepsilon}$ in this proof.

Define

$$
L_{0}:=\sqrt{\frac{\ell}{N-1}}
$$

and, for $0<\delta<1$,

$$
L_{\delta}:=L_{0}(1-\delta)
$$

We prove Proposition 2.1 by showing that for $\delta=4 \varepsilon^{1 / 2}\|\Delta U\|_{L^{\infty}}^{1 / 2}$ and $\varepsilon$ small enough so that $0<\delta<1$, the energy of any configuration $Z$ with two points, say $z_{0}$ and $z$, satisfying

$$
\operatorname{dist}\left(z_{0}, z\right)<L_{\delta}
$$

can be strictly lowered by moving $z$ to another point $z^{\prime}$ farther from $z_{0}$, namely such that

$$
\operatorname{dist}\left(z_{0}, z^{\prime}\right)=L_{0}>L_{\delta}
$$

To show this we compute the energy difference between the two configuration:2, $Z=$ $\left(z_{0}, z, z_{3}, \ldots, z_{N}\right)$ and $Z^{\prime}=\left(z_{0}, z^{\prime}, z_{3}, \ldots, z_{N}\right)$ :

$$
\begin{aligned}
H_{N}\left(z_{0}, z, z_{3}, \ldots, z_{N}\right) & -H_{N}\left(z_{0}, z^{\prime}, z_{3}, \ldots, z_{N}\right) \\
& =|z|^{2}-\left|z^{\prime}\right|^{2}+\frac{2 \ell}{N-1} w\left(z_{0}-z\right)-\frac{2 \ell}{N-1} w\left(z_{0}-z^{\prime}\right) \\
& +\frac{1}{N-1} W(Z)-\frac{1}{N-1} W\left(Z^{\prime}\right)+\frac{2 \ell}{N-1} \sum_{j=3}^{N}\left(w\left(z_{j}-z\right)-w\left(z_{j}-z^{\prime}\right)\right) \\
& +\varepsilon\left(U(z)-U\left(z^{\prime}\right)\right) .
\end{aligned}
$$

\footnotetext{
${ }^{2}$ By symmetry of $H_{N}$ we may assume that $z_{0}$ and $z$ are the first two points in the labeling.
} 
Considering the points $z_{0}, z_{3}, \ldots, z_{N}$ as fixed we write this as

$$
H_{N}(Z)-H_{N}\left(Z^{\prime}\right)=G(z)-G\left(z^{\prime}\right)+R(z)-R\left(z^{\prime}\right)+\varepsilon\left(\tilde{U}(z)-\tilde{U}\left(z^{\prime}\right)\right)
$$

where

$$
\begin{aligned}
G(z) & =\frac{2 \ell}{N-1} w\left(z_{0}-z\right)+P(z) \\
P(z) & =\frac{2}{\pi} \log |\cdot| * \mathbb{1}_{z \in B\left(z_{0}, L_{0}\right)} \\
\tilde{U}(z) & =\frac{1}{2 \pi} \log |\cdot| *\left((\Delta U) \mathbb{1}_{z \in B\left(z_{0}, L_{0}\right)}\right)
\end{aligned}
$$

and

$$
R(z)=\frac{2 \ell}{N-1} \sum_{j=3}^{N} w\left(z_{j}-z\right)+\frac{1}{N-1} W\left(z_{0}, z, z_{3}, \ldots, z_{N}\right)+|z|^{2}+\varepsilon(U(z)-\tilde{U}(z))-P(z) .
$$

We first note that $z \mapsto R(z)$ is superharmonic on $B\left(z_{0}, L_{0}\right)$. Indeed, the first two terms in (2.11) are by definition superharmonic everywhere (recall (1.17) and (1.20)) and for the combination of the last three we use that $P$ is precisely tuned in order to have

$$
-\Delta\left(|z|^{2}+\varepsilon(U(z)-\tilde{U}(z))-P(z)\right)=0
$$

in $B\left(z_{0}, L_{0}\right)$. Hence

$$
-\Delta R(z) \geq 0 \text { for } z \in B\left(z_{0}, L_{0}\right) .
$$

As a consequence $R$ reaches its minimum over $B\left(z_{0}, L_{0}\right)$ at some point $z^{\prime}$ of the boundary of the disc:

$$
R(z)-R\left(z^{\prime}\right) \geq 0
$$

with $\operatorname{dist}\left(z_{0}, z^{\prime}\right)=L_{0}$.

Next we note that

$$
\begin{aligned}
|\nabla \tilde{U}| & \leq \frac{2}{\pi}|\cdot|^{-1} *\left(|\Delta U| \mathbb{1}_{z \in B\left(z_{0}, L_{0}\right)}\right) \\
& \leq 4\|\Delta U\|_{L^{\infty}} \int_{B\left(0, L_{0}\right)} \frac{1}{|y|} d y \leq 4 L_{0}\|\Delta U\|_{L^{\infty}} .
\end{aligned}
$$

Since $\left|z-z^{\prime}\right| \leq 2 L_{0}$ we thus obtain

$$
\varepsilon\left|\tilde{U}(z)-\tilde{U}\left(z^{\prime}\right)\right| \leq 8 \varepsilon L_{0}^{2}\|\Delta U\|_{L^{\infty}} .
$$

It remains to estimate $G(z)-G\left(z^{\prime}\right)$. Since $G$ is radial around $z_{0}$ and monotonously decreasing in the radial variable on $B\left(z_{0}, L_{0}\right)$ (see Eq.(2.14) below) we can just translate the whole system to set $z_{0}=0$ and compute $G\left(L_{\delta}\right)-G\left(L_{0}\right)$. But $G$ is the potential generated by a positive point charge sitting at $z_{0}=0$ and a negative charge smeared over the ball $B\left(0, L_{0}\right)$, so

$$
G=-\log |\cdot| *\left(\frac{2 \ell}{N-1} \delta_{0}-\frac{2}{\pi} \mathbb{1}_{B\left(0, L_{0}\right)}\right) .
$$


An explicit computation using Newton's theorem shows that for $r \leq L_{0}$

$$
G(r)=2\left(L_{0}\right)^{2} \log L_{0}-\frac{2 \ell}{N-1} \log r+r^{2}-\left(L_{0}\right)^{2}
$$

and

$$
G^{\prime}(r)=-\frac{2 \ell}{N-1} \frac{1}{r}+2 r<0
$$

if $r<L_{0}$. Moreover, $G\left(L_{0}\right)=0$ and, using that $-\log (1-\delta) \geq \delta-\frac{1}{2} \delta^{2}$, we have

$$
G\left(L_{\delta}\right) \geq \frac{1}{2} \delta^{2} L_{0}^{2}
$$

Thus,

$$
G(z)-G\left(z^{\prime}\right)+\varepsilon\left(\tilde{U}(z)-\tilde{U}\left(z^{\prime}\right)\right) \geq G(z)-G\left(z^{\prime}\right)-\varepsilon\left|\tilde{U}(z)-\tilde{U}\left(z^{\prime}\right)\right|>0
$$

provided

$$
\frac{1}{2} \delta^{2} L_{0}^{2}-8 \varepsilon L_{0}^{2}\|\Delta U\|_{L^{\infty}}>0, \quad \text { i.e. } \quad \delta>4 \varepsilon^{1 / 2}\|\Delta U\|_{L \infty}^{1 / 2} .
$$

In view of (2.10) and (2.12) we thus have

$$
H_{N}(Z)>H_{N}\left(Z^{\prime}\right)
$$

Hence (2.9) with $\delta$ as in (2.15) cannot hold for a minimizing configuration, so (2.2) must hold in such a configuration and the proof of Proposition 2.1 is complete.

\section{Applications to GibBs states at small temperature}

In this section we deduce incompressibility bounds for the Gibbs state (1.15) at $T=N^{-1}$ from the zero-temperature results of the previous section. The main step is to bound from below the energy in a regular potential $U$ with bounded derivatives, which should be thought of as a truncation of the physical potential $V$ appearing in Theorem 1.1, see Section 3.2 below.

Proposition 3.1 (Energy lower bounds in truncated potentials).

Let $\mu_{N}$ be defined as in (1.15), let $U \in C^{2}\left(\mathbb{R}^{2}\right)$ be such that $U, \Delta U \in L^{\infty}\left(\mathbb{R}^{2}\right)$. For any $N$ large enough and $\varepsilon>0$ small enough there exists a probability density $\rho \in L^{1}\left(\mathbb{R}^{2}\right)$ satisfying

$$
\rho \leq \frac{4}{\pi \ell}\left(1+8 \varepsilon^{1 / 2}\|\Delta U\|_{L^{\infty}}^{1 / 2}\right)\left(1-N^{-1}\right)
$$

such that, for a constant $C<\infty$,

$$
\int_{\mathbb{R}^{2}} U \mu_{N}^{(1)} \geq \int_{\mathbb{R}^{2}} U \rho-\frac{C}{\varepsilon N}(1+\log N)-C N^{-1 / 2}\|\nabla U\|_{L^{\infty}}
$$

Note the necessary adjustment of $\varepsilon$ in applications: the smaller it is, the closer the density bound (3.1) is from the desired one, but the larger the error term in (3.2). We prove this result in the next Section 3.1 and deduce from it our main Theorem 1.1 in Section 3.2 . 
3.1. Small temperature limit. The proof consists of upper and lower bounds to the free energy

$$
F_{N}^{\varepsilon}:=\inf \left\{\mathcal{F}_{N}^{\varepsilon}[\mu], \quad \mu \in \mathcal{P}\left(\mathbb{R}^{2 N}\right)\right\}
$$

where the free energy functional on the space $\mathcal{P}\left(\mathbb{R}^{2 N}\right)$ of probability measures on $\mathbb{R}^{2}$ is

$$
\mathcal{F}_{N}^{\varepsilon}[\mu]:=\int_{\mathbb{R}^{2 N}} H_{N}^{\varepsilon}\left(Z_{N}\right) \mu\left(Z_{N}\right) d Z_{N}+N^{-1} \int_{\mathbb{R}^{2 N}} \mu \log \mu
$$

with the perturbed Hamiltonian (2.1). It is well-known that the infimum is achieved by the Gibbs measure

$$
\mu_{N}^{\varepsilon}\left(Z_{N}\right)=\frac{1}{\mathcal{Z}_{N}^{\varepsilon}} \exp \left(-N H_{N}^{\varepsilon}\left(Z_{N}\right)\right) \in \mathcal{P}\left(\mathbb{R}^{2 N}\right)
$$

where the partition function satisfies $-N^{-1} \log \mathcal{Z}_{N}^{\varepsilon}=F_{N}^{\varepsilon}$. The perturbation by the onebody potential $\varepsilon U$ of the original Hamiltonian (1.16) will allow us to deduce density bounds from free-energy estimates, in the spirit of the Feynman-Hellmann principle.

Free energy upper bound. By the variational principle we have

$$
F_{N}^{\varepsilon} \leq \mathcal{F}_{N}^{\varepsilon}\left[\mu_{N}\right]=F_{N}^{0}+\varepsilon N \int_{\mathbb{R}^{2}} U \mu_{N}^{(1)}
$$

To obtain an upper bound on $F_{N}^{0}$ we use a trial state which is a regularization, over a length scale $\eta$ to be later optimized over, of a ground state configuration $Z_{N}^{0}=\left(z_{1}^{0}, \ldots, z_{N}^{0}\right)$ for $H_{N}$ :

$$
\mu_{N}^{t}\left(z_{1}, \ldots, z_{N}\right):=\left(\frac{1}{\pi \eta^{2}}\right)^{N} \mathbb{1}_{z_{1} \in B\left(z_{1}^{0}, \eta\right)} \otimes \ldots \otimes \mathbb{1}_{z_{N} \in B\left(z_{N}^{0}, \eta\right)} .
$$

Note that this probability measure is not symmetric under particle exchange, but that is of no concern because the symmetry of the Hamiltonian implies that the infimum in (3.3) is the same with or without symmetry constraint. The entropy of $\mu_{N}^{t}$ is

$$
S=-\int_{\mathbb{R}^{2 N}} \mu_{N}^{t} \log \mu_{N}^{t}=N \log \left(\pi \eta^{2}\right)
$$

and since the temperature is $T=N^{-1}$ the contribution $-T S$ to the free energy is $-\log \left(\pi \eta^{2}\right)$. For the energetic part we note that

$\int_{\mathbb{R}^{2 N}} H_{N}\left(Z_{N}\right) \mu_{N}^{t}\left(Z_{N}\right) d Z_{N} \leq \sum_{j=1}^{N} \frac{1}{\pi \eta^{2}} \int_{B\left(z_{j}^{0}, \eta\right)}|z|^{2} d z+\frac{2 \ell}{N-1} \sum_{1 \leq i<j \leq N} w\left(z_{i}^{0}-z_{j}^{0}\right)+\frac{1}{N-1} W\left(Z_{N}^{0}\right)$,

because superharmonicity in each variable of the function

$$
\left(z_{1}, \ldots, z_{N}\right) \mapsto \frac{2 \ell}{N-1} \sum_{1 \leq i<j \leq N} w\left(z_{i}-z_{j}\right)+\frac{1}{N-1} W\left(Z_{N}\right)
$$

implies that it must decrease upon taking an average over balls centered at the $z_{i}^{0}$ 's. For the one-body term we have, integrating in polar coordinates

$$
\frac{1}{\pi \eta^{2}} \int_{B\left(z_{j}^{0}, \eta\right)}|z|^{2} d z=\frac{1}{\pi \eta^{2}} \int_{B(0, \eta)}\left|z+z_{j}^{0}\right|^{2} d z=\left|z_{j}^{0}\right|^{2}+\frac{1}{\pi \eta^{2}} \int_{B(0, \eta)}|z|^{2} d z=\left|z_{j}^{0}\right|^{2}+\frac{\eta^{2}}{2}
$$


All in all we thus have, for $N$ large enough,

$$
\begin{aligned}
F_{N}^{\varepsilon} & \leq H_{N}\left(Z_{N}^{0}\right)+\varepsilon N \int_{\mathbb{R}^{2}} U \mu_{N}^{(1)}-\log (\pi)-\log \eta^{2}+N \frac{\eta^{2}}{2} \\
& \leq \min _{\mathbb{R}^{2 N}} H_{N}+\varepsilon N \int_{\mathbb{R}^{2}} U \mu_{N}^{(1)}+C(\log N+1) .
\end{aligned}
$$

where we chose $\eta=(2 N)^{-1 / 2}$ to optimize the error.

Free energy lower bound. To bound $F_{N}^{\varepsilon}$ from below we start with the entropy term: we first set

$$
\nu=\pi^{-1} \exp \left(-|z|^{2}\right)
$$

Then

$$
\begin{aligned}
\int_{\mathbb{R}^{2 N}} \mu_{N}^{\varepsilon} \log \mu_{N}^{\varepsilon} & =\int_{\mathbb{R}^{2 N}} \mu_{N}^{\varepsilon} \log \frac{\mu_{N}^{\varepsilon}}{\nu^{\otimes N}}+\int_{\mathbb{R}^{2 N}} \mu_{N}^{\varepsilon} \log \nu^{\otimes N} \\
& \geq \int_{\mathbb{R}^{2 N}} \mu_{N}^{\varepsilon} \log \nu^{\otimes N} \\
& =N \int_{\mathbb{R}^{2}}\left(\mu_{N}^{\varepsilon}\right)^{(1)} \log \nu=-N \int_{\mathbb{R}^{2}}|z|^{2}\left(\mu_{N}^{\varepsilon}\right)^{(1)}-N \log \pi
\end{aligned}
$$

using positivity of the relative entropy of two probability measures. Adding the energy term we obtain

$$
\begin{aligned}
F_{N}^{\varepsilon} & =\int_{\mathbb{R}^{2 N}} H_{N}^{\varepsilon}\left(Z_{N}\right) \mu_{N}^{\varepsilon}\left(Z_{N}\right) d Z_{N}+N^{-1} \int_{\mathbb{R}^{2 N}} \mu_{N}^{\varepsilon} \log \mu_{N}^{\varepsilon} \\
& \geq \int_{\mathbb{R}^{2 N}}\left(H_{N}^{\varepsilon}\left(Z_{N}\right)-N^{-1} \sum_{j=1}^{N}\left|z_{j}\right|^{2}\right) \mu_{N}^{\varepsilon}\left(Z_{N}\right) d Z_{N}-\log \pi \\
& \geq \min _{Z_{N} \in \mathbb{R}^{2 N}}\left\{H_{N}^{\varepsilon}\left(Z_{N}\right)-N^{-1} \sum_{j=1}^{N}\left|z_{j}\right|^{2}\right\}-\log \pi \\
& \geq \min _{Z_{N} \in \mathbb{R}^{2 N}} H_{N}^{\varepsilon}\left(Z_{N}\right)-C=H_{N}^{\varepsilon}\left(Z_{N}^{\varepsilon}\right)-C \\
& \geq H_{N}\left(Z_{N}^{\varepsilon}\right)+N \varepsilon \int_{\mathbb{R}^{2}} U(z) \rho_{0}^{\varepsilon}-C \\
& \geq \min _{\mathbb{R}^{2 N}} H_{N}+N \varepsilon \int_{\mathbb{R}^{2}} U \rho_{0}^{\varepsilon}-C
\end{aligned}
$$

where (3.9) follows from a simple estimate on the value of $\sum_{j=1}^{N}\left|z_{j}\right|^{2}$ at a minimum configuration of the curly brace in (3.8) (see similar computations in [18, Section 3]), and $\rho_{0}^{\varepsilon}$ is the empirical measure of a minimizing configuration $Z_{N}^{\varepsilon}$ for $H_{N}^{\varepsilon}$ defined in (2.3).

Density bound. Combining (3.7) and (3.10) we obtain

$$
\int_{\mathbb{R}^{2}} U \mu_{N}^{(1)} \geq \int_{\mathbb{R}^{2}} U \rho_{0}^{\varepsilon}-\frac{C}{\varepsilon N}(1+\log N)
$$


and we may apply Corollary 2.2 to $\rho_{0}^{\varepsilon}$ to obtain a probability density $\rho:=\tilde{\rho}_{0}^{\varepsilon}$ satisfying the requirements of Proposition 3.1 and such that (3.2) holds, i.e.,

$$
\int_{\mathbb{R}^{2}} U \mu_{N}^{(1)} \geq \int_{\mathbb{R}^{2}} U \rho-\frac{C}{\varepsilon N}(1+\log N)-C N^{-1 / 2}\|\nabla U\|_{L^{\infty}}
$$

3.2. Conclusion of the proof of Theorem 1.1. To conclude the proof of our main theorem we proceed as in [18, Section 4.1]. We pick some (sequence of) correlation factor(s) $F \in \mathcal{B}^{N}$, construct the corresponding state(s) $\Psi \in \mathcal{L}_{\ell}^{N}$, normalized in $L^{2}$, and Gibbs measure (s) $\mu_{N}$. We also pick a large constant $B$ (to be tuned later on) and define the truncated potential

$$
V_{B}(\mathbf{x}):=\min \{V(\mathbf{x}), B\} .
$$

Thanks to (1.10), this potential is constant outside of some ball centered at the origin and satisfies the assumptions of Proposition 3.1. We may thus apply this result with $U=V_{B}$ and the correlation factor $F$ at hand. In view of (1.15) we have

$$
\mu_{N}^{(1)}(z)=(N-1) \rho_{\Psi}(\sqrt{N-1} z)
$$

and the proposition implies that there exists a $\rho=\rho_{F}$ of unit $L^{1}$ norm satisfying

$$
0 \leq \rho_{F} \leq M_{\varepsilon, N}:=\frac{4}{\pi \ell}\left(1+8 \varepsilon^{1 / 2}\left\|\Delta V_{B}\right\|_{L^{\infty}}^{1 / 2}\right)\left(1-N^{-1}\right)
$$

such that

$$
\begin{aligned}
\mathcal{E}_{N}\left[\Psi_{F}\right] & \geq(N-1) \int_{\mathbb{R}^{2}} V_{B}(\mathbf{x}) \rho_{\Psi_{F}}(\sqrt{N-1} \mathbf{x}) d \mathbf{x} \\
& \geq \int_{\mathbb{R}^{2}} V_{B} \rho_{F}-\frac{C}{\varepsilon N}(1+\log N)-C N^{-1 / 2}\left\|\nabla V_{B}\right\|_{L^{\infty}} \\
& \geq \inf \left\{\int_{\mathbb{R}^{2}} V_{B} \rho, 0 \leq \rho \leq M_{\varepsilon, N}, \int_{\mathbb{R}^{2}} \rho=1\right\}-\frac{C}{\varepsilon N}(1+\log N)-C N^{-1 / 2}\left\|\nabla V_{B}\right\|_{L^{\infty}}
\end{aligned}
$$

Passing then to the limit $N \rightarrow \infty$ at fixed $\varepsilon$ and $B$ we obtain

$$
\liminf _{N \rightarrow \infty} \mathcal{E}_{N}\left[\Psi_{F}\right] \geq \inf _{\rho}\left\{\int_{\mathbb{R}^{2}} V_{B} \rho, 0 \leq \rho \leq \frac{4}{\pi \ell}\left(1+8 \varepsilon^{1 / 2}\left\|\Delta V_{B}\right\|_{L^{\infty}}^{1 / 2}\right), \int_{\mathbb{R}^{2}} \rho=1\right\}
$$

and, since the right-hand side no longer depends on $F$, we have

$$
\liminf _{N \rightarrow \infty} E(N) \geq \inf _{\rho}\left\{\int_{\mathbb{R}^{2}} V_{B} \rho, 0 \leq \rho \leq \frac{4}{\pi \ell}\left(1+8 \varepsilon^{1 / 2}\left\|\Delta V_{B}\right\|_{L^{\infty}}^{1 / 2}\right), \int_{\mathbb{R}^{2}} \rho=1\right\} .
$$

We may then pass to the limit $\varepsilon \rightarrow 0$ at fixed $B$ :

$$
\liminf _{N \rightarrow \infty} E(N) \geq \inf _{\rho}\left\{\int_{\mathbb{R}^{2}} V_{B} \rho, 0 \leq \rho \leq \frac{4}{\pi \ell}, \int_{\mathbb{R}^{2}} \rho=1\right\}
$$

and finally to the limit $B \rightarrow \infty$, which yields

$$
\liminf _{N \rightarrow \infty} E(N) \geq E_{V}(\ell / 4)
$$

as desired. We have used some continuity properties of the bath-tub energy (1.11) as a function of the upper bound on the admissible trial states and the cut-off of the potential. 
N. ROUGERIE AND J. YNGVASON

These follow easily from the explicit formula for the minimum bath-tub energy see 10 , Theorem 1.14]. Note in particular that for $B$ large enough, the bathtub energy in $V_{B}$ is in fact equal to the bathtub energy in $V$.

\section{REFERENCES}

[1] G. Anderson, A. Guionnet, And O. Zeitouni, An introduction to random matrices, Cambridge University Press, 2010.

[2] O. Ciftú́, Monte Carlo study of Bose Laughlin wave function for filling factors 1/2, 1/4 and 1/6, Europhys. Lett., 74 (2006), pp. 486-492.

[3] F. J. Dyson, Statistical theory of the energy levels of a complex system. part I, J. Math. Phys., 3 (1962), pp. $140-156$.

[4] P. Forrester, Log-gases and random matrices, London Mathematical Society Monographs Series, Princeton University Press, 2004.

[5] J. Ginibre, Statistical ensembles of complex, quaternion, and real matrices, J. Math. Phys., 6 (1965), pp. $440-449$.

[6] S. GiRvin, Introduction to the fractional quantum Hall effect, Séminaire Poincaré, 2 (2004), pp. 54-74.

[7] R. B. Laughlin, Anomalous quantum Hall effect: An incompressible quantum fluid with fractionally charged excitations, Phys. Rev. Lett., 50 (1983), pp. 1395-1398.

[8] —, Elementary theory : the incompressible quantum fluid, in The quantum Hall effect, R. E. Prange and S. E. Girvin, eds., Springer, Heidelberg, 1987.

[9] M. Lewin and R. Seiringer, Strongly correlated phases in rapidly rotating Bose gases, J. Stat. Phys., 137 (2009), pp. 1040-1062.

[10] E. H. Lieb And M. Loss, Analysis, vol. 14 of Graduate Studies in Mathematics, American Mathematical Society, Providence, RI, second ed., 2001.

[11] M. Mehta, Random matrices. Third edition, Elsevier/Academic Press, 2004.

[12] T. Papenbrock And G. F. Bertsch, Rotational spectra of weakly interacting Bose-Einstein condensates, Phys. Rev. A, 63 (2001), p. 023616.

[13] M. Petrache And S. Serfaty, Next order asymptotics and renormalized energy for Riesz interactions, ArXiv e-prints, (2014).

[14] S. Rota-Nodari And S. SERfaty, Renormalized energy equidistribution and local charge balance in 2d Coulomb systems, ArXiv e-prints, (2013).

[15] N. Rougerie And S. SERFATy, Higher dimensional Coulomb gases and renormalized energy functionals, ArXiv e-prints, (2013).

[16] N. Rougerie, S. Serfaty, And J. Yngvason, Quantum Hall phases and plasma analogy in rotating trapped bose gases, J. Stat. Phys., (2013).

[17] —, Quantum Hall states of bosons in rotating anharmonic traps, Phys. Rev. A, 87 (2013), p. 023618.

[18] N. Rougerie And J. Yngvason, Incompressibility estimates for the Laughlin phase, Comm. Math. Phys., (2014).

[19] E. Sandier and S. Serfaty, 2D Coulomb Gases and the Renormalized Energy, ArXiv e-prints, (2012).

[20] H. Störmer, D. Tsui, And A. Gossard, The fractional quantum Hall effect, Rev. Mod. Phys., 71 (1999), pp. S298-S305.

[21] S. Trugman and S. Kivelson, Exact results for the fractional quantum Hall effect with general interactions, Phys. Rev. B, 31 (1985), p. 5280.

Université Grenoble 1 \& CNRS, LPMMC (UMR 5493), B.P. 166, F-38 042 Grenoble, France E-mail address: nicolas.rougerie@grenoble.cnrs.fr

Fakultät für Physik, Universität Wien, Boltzmanngasse 5, 1090 Vienna, Austria \& Erwin Schrödinger Institute for Mathematical Physics, Boltzmanngasse 9, 1090 Vienna, Austria.

E-mail address: jakob.yngvason@univie.ac.at 